\title{
Path-dependent Self-assembly of Magnetic Anisotropic Colloidal Peanuts
}

\author{
Md. Arif Kamal, ${ }^{\dagger} \S$ Andrei V. Petukhov, ${ }^{\ddagger}$ and Antara Pal ${ }^{*, \Phi}$ \\ †Centre Interdisciplinaire de Nanoscience de Marseille (CINaM), CNRS, Aix Marseille \\ University, Marseille, France \\ $\ddagger$ Van't Hoff Laboratory for Physical and Colloid Chemistry, Utrecht University, The \\ Netherlands \\ 【Division of Physical Chemistry, Department of Chemistry, Lund University, Lund, \\ Sweden \\ $\S$ Current Address: Division of Physical Chemistry, Department of Chemistry, Lund \\ University, Lund, Sweden \\ E-mail: antara.pal@fkem1.lu.se
}


a

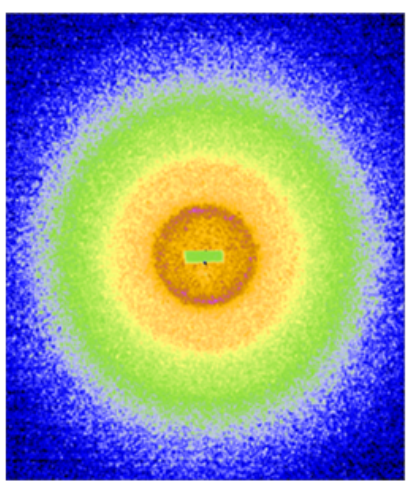

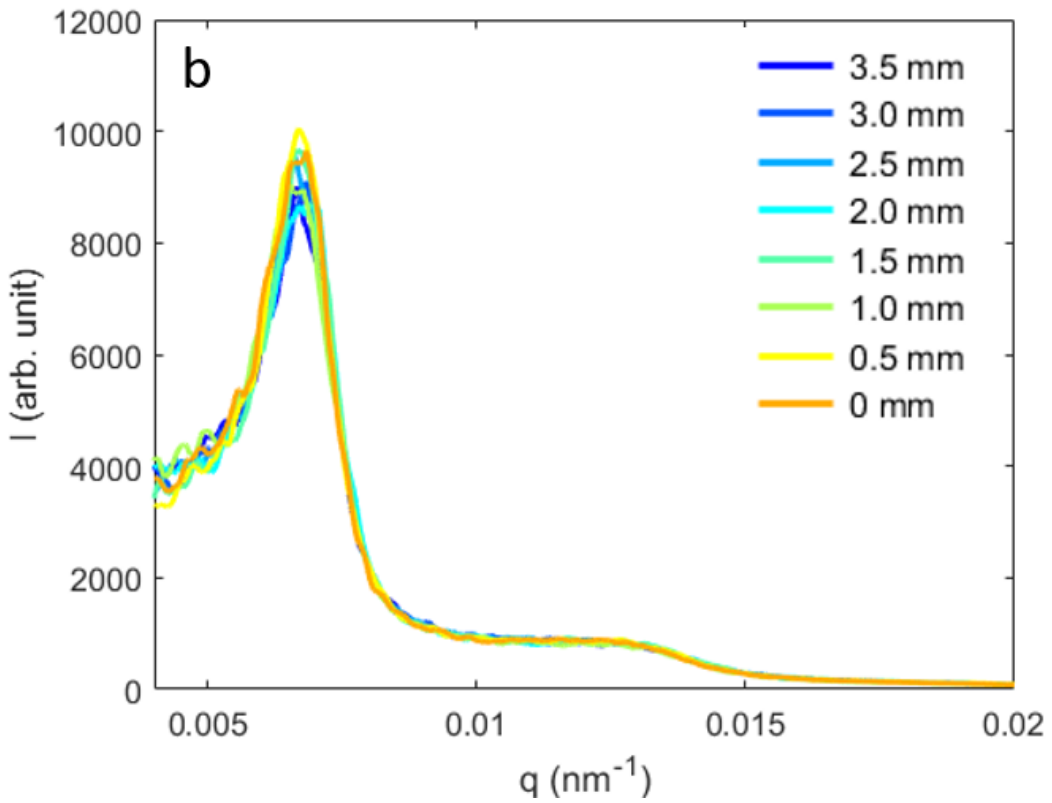

Figure S1: (a) A representative diffraction pattern for the isotropic phase which was formed in absence of the external field. (b) Variation of the radial intensity profiles for different heights $(\mathrm{Z})$ in absence of the field. 